\title{
Avaliação do desempenho de estudantes de Medicina em Neurologia utilizando metodologias ativas
}

\author{
Evaluation of the performance of medical students in Neurology using active \\ methodologies \\ Evaluación del desempeño de estudiantes de Medicina en Neurología utilizando \\ metodologías activas
}

\author{
Celina Cláudia Israel Sefer ${ }^{1 \star}$, Lorena Fecury Tavares², Rafael de Azevedo Silva², Marcia \\ Bitar Portella², Nara Macedo Botelho'1.
}

\section{RESUMO}

Objetivo: Avaliar o desempenho dos alunos do curso de Medicina no módulo de semiologia neurológica utilizando metodologias ativas Métodos: Trata-se de estudo prospectivo, transversal, e com abordagem quantitativa, com 42 estudantes de Medicina de uma escola médica privada, avaliados antes (pré-teste) e depois de cursarem o módulo de semiologia neurológica. Após o módulo realizaram um pós-teste (1) com questões de múltipla escolha e o OSCE, a fim de definir desempenho a curto prazo. Após 1 ano realizaram pós-teste 2 e OSCE 2 (nos mesmos moldes dos primeiros), averiguando o conhecimento a médio prazo. $O$ estudo foi aprovado pelo Comitê de Ética em Pesquisa. Resultados: Houve diferença significativa entre as notas do pré-teste e pós-teste 1, porém sem correlação significativa entre elas. Observou-se um bom desempenho no aprendizado a curto prazo, com equivalência nos testes teóricos e no OSCE. Na avaliação de médio prazo observou-se redução do conhecimento teórico, porém mantendo bom desempenho nas habilidades práticas. Conclusão: Conclui-se que houve um incremento das médias entre pré-teste e pós teste-1, um bom desempenho no aprendizado a curto prazo, com equivalência na esferas cognitiva e prática. $\mathrm{Na}$ avaliação de médio prazo observou-se redução do conhecimento teórico, porém mantendo bom desempenho nas habilidades práticas.

Palavras-chave: Educação médica, Semiologia neurológica, avaliação estudante de Medicina

\begin{abstract}
Objective: To evaluate the performance of medical students in the neurological semiology clerkship using active Methods: This was a prospective, cross-sectional, quantitative study with 42 medical students from a private medical school, evaluated before (pre-test) and after attending the neurological semiology module. After the clerkship they performed a post-test (1) with multiple-choice questions and the OSCE in order to define short-term performance. After 1 year they performed post-test 2 and OSCE 2 (in the same way as the first ones), checking the knowledge in the medium term. The study was approved by the Research Ethics Committee. Results: There was a significant difference between the pre-test and post-test 1 scores, but with no significant correlation between them. Good performance in short-term learning, with equivalence in theoretical tests and in the OSCE, was observed. In the mid-term evaluation it was observed a reduction of theoretical knowledge, but maintaining good performance in practical skills. Conclusion: It was concluded that there was an increase in the means between pre-test and post-test 1, a good performance in short-term learning, with equivalence in the cognitive and practical spheres. In the mid-term evaluation it was observed a reduction of theoretical knowledge, but maintaining good performance in practical skills.
\end{abstract}

Key words: Medical education, neurological semiology, medical student evaluation.

${ }^{1}$ Universidade do Estado do Pará (UEPA), Belém-Pará * E-mail: celinaisrael@yahoo.com.br

2 Centro Universitário Metropolitano da Amazônia (UNIFAMAZ), Belém-Pará. 


\section{RESUMEN}

Objetivo: Evaluar el desempeño de los alumnos del curso de Medicina en el módulo de semiología neurológica utilizando metodologías activas. Métodos: Se trata de un estudio prospectivo, transversal, y con abordaje cuantitativo, con 42 estudiantes de Medicina de una escuela médica privada, evaluados antes (pretest) y después de cursar el módulo de semiología neurológica. Después de que el módulo de llevar a cabo un post-test (1) con preguntas de elección múltiple y la OSCE con el fin de establecer el rendimiento a corto plazo. Después de 1 año realizaron post-test 2 y OSCE 2 (en los mismos moldes de los primeros), averiguando el conocimiento a medio plazo. El estudio fue aprobado por el Comité de Ética en Investigación. Resultados: Hubo diferencia significativa entre las notas del pre-test y post-test 1, pero sin correlación significativa entre ellas. Se observó un buen desempeño en el aprendizaje a corto plazo, con equivalencia en las pruebas teóricas y en el OSCE. En la evaluación de mediano plazo se observó reducción del conocimiento teórico, pero manteniendo buen desempeño en las habilidades prácticas. Conclusión: Se concluye que hubo un incremento de las medias entre pre-test y post test-1, un buen desempeño en el aprendizaje a corto plazo, con equivalencia en las esferas cognitiva y práctica. En la evaluación de mediano plazo se observó reducción del conocimiento teórico, pero manteniendo buen desempeño en las habilidades prácticas.

Palabras clave: educacion medica, semiología neurológica, evaluación estudiante de medicina.

\section{INTRODUÇÃO}

As metodologias ativas de aprendizado representam atualmente um dos modelos mais aceitos para 0 currículo na educação médica, visto que incentivam o aluno a integrar o conhecimento básico, clínico e habilidades. Tais metodologias baseiam-se na aprendizagem por descoberta, construindo o conhecimento por meio de experiências significativas (CYRINO EG e TORALLES-PEREIRA ML, 2004; VUMA S e SA B, 2015)

Frente a esse novo formato de ensino surge grande dificuldade em encontrar formas de avaliação que sejam compatíveis com a filosofia da Aprendizagem Baseada em Problemas - metodologia de aprendizado, também chamado de PBL (Problem Based Learning) nas escolas médicas. (VUMA et al., 2015).

Em 1990, George Miller propôs um modelo hierárquico de avaliação das competências clínicas, sugerindo que diferentes métodos são necessários para a avaliação adequada dos diferentes níveis de competência: a base se refere ao conhecimento ("saber"); o segundo nível envolve a habilidade de aplicar o conhecimento ("saber como faz"); o terceiro reflete a habilidade de agir ("mostrar como faz"); e o quarto nível se refere à prática em situações clínicas reais ("fazer").

Atualmente, a avaliação dos alunos do curso de Medicina é direcionada para a avaliação do conhecimento, das habilidades clínicas e de comunicação. A maneira mais frequente de avaliar o conhecimento é de forma escrita, seja com questões de múltipla escolha, questões dissertativas, e suas variantes. Já o desempenho pode ser avaliado de duas maneiras diferentes: in vitro, em ambientes de simulação padronizados, e in vivo, em condições reais. (AL-WARDY, 2010) (ISMAIL, 2016).

No contexto das avaliações em ambiente controlado, destaca-se o OSCE (Objective Structured Clinical Examination), criado em 1975 por pesquisadores da Universidade de Dundee (Reino Unido) (VUMA et al., 2015), ferramenta padronizada para avaliar de forma objetiva as competências clínicas, incluindo anamnese, exame físico, habilidades de comunicação, interpretação de dados, entre outros, com confiabilidade. Há relatos de sua utilização em diversas especialidades: reumatologia, radiologia, urologia, terapia intensiva e cirurgia geral. A experiência na aplicação do OSCE na área de neurologia, no entanto, é limitada, pois existem poucos trabalhos publicados (poucos dados) analisando a aplicação do OSCE de neurologia clínica na graduação médica e na residência em neurologia. (ISMAIL, 2016) (LUKAS et al., 2012).

As doenças neurológicas representam uma parcela importante das patologias que trazem alta taxa de morbidade e mortalidade no mundo, e com o envelhecimento populacional torna-se cada vez mais comum o manejo de pacientes com doença neurológica por médicos generalistas. A correta identificação de sinais e 
sintomas é o ponto de partida para se alcançar o diagnóstico, assim é crítico que futuros médicos se tornem proficientes na realização do exame físico neurológico (ROZE, 2016) (SAFDIEH et al., 2011)

Tão importante quanto oferecer a oportunidade de aprendizado aos alunos, é avaliar a retenção desse conhecimento e aquisição das habilidades necessárias, a curto, médio e longo prazo. Sabe-se que provas e avaliações são motivos de grande estresse e ansiedade nos alunos, mas os estimulam a intensificar os estudos e o treino das habilidades. Dessa forma é de suma importância não apenas estimular a aquisição de conhecimento durante as aulas, mas também avaliar frequentemente o seu desempenho. (KHAN, 2011)

Pela falta de dados sobre o desempenho dos alunos de medicina no módulo de semiologia neurológica, e pela necessidade de um feedback sobre a efetividade do método empregado, a fim de adequar as práticas às exigências das novas diretrizes curriculares nacionais para a formação de um bom médico, torna-se premente reunir informações sobre o aprendizado dos alunos, em habilidades clínicas, no módulo de neurologia.

Logo, o objetivo da presente pesquisa é avaliar o desempenho dos alunos do curso de medicina no módulo de semiologia neurológica utilizando metodologias ativas.

\section{MÉTODOS}

\section{Questões éticas}

A pesquisa foi aprovada pelo Comitê de Ética em Pesquisa (CEP) do Centro Universitário Metropolitano da Amazônia (UNIFAMAZ), obedecendo à resolução n 466/12 do Conselho Nacional de Saúde pelo número: CAAE 64526016.6.0000.5084.

Todos os acadêmicos incluídos na pesquisa foram estudados segundo os preceitos éticos após a assinatura do Termo de Consentimento Livre e Esclarecido (TCLE), garantindo anonimato e segurança aos participantes.

\section{Tipo de estudo}

Foi realizado um estudo prospectivo, transversal, e com abordagem quantitativa, com 42 estudantes de Medicina de uma instituição de ensino superior (IES) privada (UNIFAMAZ), no período de setembro de 2016 a setembro de 2017.

\section{Local do estudo}

O estudo foi realizado no laboratório de Habilidades Clínicas do Centro Universitário Metropolitano da Amazônia.

\section{Sujeitos da pesquisa}

Foram avaliados 42 alunos do curso de Medicina, que concordaram em participar após esclarecimentos dos seus objetivos, métodos, riscos e benefícios, e que estavam regularmente matriculados no $4^{\circ}$ semestre do curso na ocasião do início da pesquisa. Foram excluídos aqueles que desistiram do curso ao longo do estudo e os que não completaram todas as etapas das avaliações necessárias ou os que já tinham algum conhecimento prévio de neurologia em realização de outro curso.

A seleção da amostra foi feita por um convite direto da pesquisadora aos estudantes (amostra aleatória simples). Do universo de 46 alunos matriculados naquele semestre, 42 foram incluídos com sucesso.

\section{Coleta de dados}

A coleta de dados foi realizada em dois momentos diferentes: o primeiro enquanto cursaram o quarto semestre do curso, e o segundo um ano após, enquanto cursavam o sexto semestre. O módulo de semiologia 
neurológica é regularmente ofertado no $4^{\circ}$ semestre do Curso de Medicina da UNIFAMAZ, em conjunto com o módulo de semiologia do aparelho locomotor. Dessa forma a primeira avaliação representava o aprendizado a curto prazo, e a segunda, representava o aprendizado a médio prazo.

O estudo teve o seguinte delineamento: uma semana antes do início do módulo de neurologia os alunos foram submetidos a um teste teórico a fim de avaliar o conhecimento prévio (pré-teste) com 10 questões de múltipla escolha contemplando aspectos práticos dos conteúdos ministrados, distribuídos nas seguintes áreas de conhecimento: coordenação motora, nervos cranianos, reflexos, motricidade automática, motricidade voluntária, sensibilidade e sinais neurológicos especiais.

Cada questão trazia 4 opções de resposta (de A a D), e nível de dificuldade com $25 \%$ de questões classificadas como fáceis, $50 \%$ como nível médio, e $25 \%$ como difíceis. O módulo teve duração aproximada de 4 meses, com carga horária de 8 horas semanais e contou com a participação de 4 docentes da IES. O conteúdo da semiologia neurológica abordou os principais pontos do roteiro do exame, simplificado para o generalista. As aulas iniciavam com uma breve explanação do professor sobre o roteiro, acompanhada da demonstração prática do exame físico específico. A seguir os alunos iam para a prática interpares em ambiente protegido, quando os professores realizavam a avaliação formativa dos alunos, fornecendo o feedback ao final de cada aula.

Ao final do módulo foi realizado um pós-teste (pós-teste 1) nos mesmos moldes do pré-teste, com o mesmo número de questões e nível de dificuldade. No mesmo dia, após a avaliação teórica, realizaram o OSCE-1, constituído de 4 estações, com casos clínicos breves abordando o exame dos nervos cranianos, a avaliação da motricidade e reflexos, sensibilidade e testes especiais, e o exame da coordenação motora.

Na segunda avaliação, um ano após, foi realizado o pós-teste 2, com 10 questões de múltipla escolha, e - OSCE- 2 ambos obedecendo aos mesmos padrões e nível de dificuldade dos anteriores. No período entre a primeira avaliação e a segunda, esses acadêmicos não tiveram contato com conteúdo de semiologia neurológica ou neurologia clínica pelo currículo da IES.

A elaboração de todas as questões dos testes teóricos, casos clínicos e checklists do OSCE, contou com a orientação de um grupo de 4 professores da instituição, incluindo o pesquisador principal, obedecendo as exigências de contextualização e objetivos educacionais. Posteriormente esse conteúdo foi revisado pela equipe de assessoria pedagógica do curso de Medicina da UNIFAMAZ. Somente após essa última revisão foram utilizadas na avaliação dos alunos. Os testes teóricos e os OSCE's também foram supervisionados, realizados e corrigidos por esse mesmo grupo de professores.

\section{Método Estatístico}

Para analisar dados de avaliações aplicada a $n=42$ estudantes de Medicina foram aplicados métodos estatísticos descritivos e inferenciais.

Para avaliar a Hipótese 1 (considerando as médias das notas há real diferença entre pré-teste e pós-teste 1), a Hipótese 2a (considerando as médias das notas há real diferença entre o pós-teste 1 e o pós-teste 2) e a Hipótese 3b (existe diferença entre o pós -teste 1 e o OSCE 1) foi aplicado o teste t de Student para amostras pareadas. Para avaliar a Hipótese $2 \mathrm{~b}$ (nos módulos do OSCE o número de acertos difere entre OSCE- 1 e OSCE- 2) foi aplicado o teste Binomial. Para avaliar a hipótese 3a (existe real correlação entre os pós-testes e os OSCEs) foi aplicada a Correlação Linear de Pearson. Para avaliar foi aplicado o teste t de Student para amostras pareadas. Para avaliar a normalidade das variáveis quantitativas foi aplicado o teste de Normalidade de D'Agostino-Pearson.

Foi previamente fixado o nível de significância alfa $=0.05$ para rejeição da hipótese de nulidade. 0 processamento estatístico foi realizado no programa BioEstat versão 5.3 e o planejamento da análise de dados foi realizado na tecnologia SAM (Statistical Analysis Model). 


\section{RESULTADOS}

Os educadores médicos têm sido desafiados a desenvolver avaliações que sejam relevantes para o trabalho do médico e também que abranjam o espectro do desempenho esperado. Neste estudo, utilizou-se testes teóricos de múltipla escolha e o OSCE como ferramentas avaliativas para os domínios da semiologia neurológica, em 2 momentos diferentes.

Ao se avaliar o desempenho dos sujeitos no pré-teste e pós-teste 1 observou-se que houve diferença significativa entre as notas, com evolução nas médias, porém não houve correlação significativa entre elas. Isso pode ter explicações diferentes: ou os acadêmicos adquiriram mais conhecimento sobre os assuntos abordados ou houve certa displicência ao realizar o pré-teste, já que os alunos poderiam valer-se da sorte ao marcar as questões.

\section{Desempenho no pré e pós-teste 1}

A média no pré-teste foi de 4.0 pontos e no pós-teste 1 foi de 7.1 pontos, confirmado pelo teste t de Student (p-valor $\left.<0.0001^{*}\right)$.

Observou-se ainda que a tendência crescente no pré-teste não foi a mesma no pós-teste, por esse motivo não existiu real correlação. Contudo, há real diferença entre as médias que evoluíram de 4.0 (pré-teste) para 7.1 (pós-teste) (Tabela 1).

Tabela 1 - Avaliação do Pré-teste e Pós-teste 1 de n=42 estudantes de medicina. Belém/PA, ano 2018.

\begin{tabular}{lcc}
\hline & $\begin{array}{c}\text { Pré-teste } \\
(\mathbf{n}=\mathbf{4 2})\end{array}$ & $\begin{array}{c}\left(\mathbf{1}^{\circ}\right) \text { Pós-teste } \\
(\mathbf{n}=\mathbf{4 2})\end{array}$ \\
\hline Mínimo & 1.0 & 3.0 \\
Máximo & 8.0 & 10.0 \\
Mediana & 4.0 & 7.0 \\
Primeiro Quartil & 3.0 & 6.0 \\
Terceiro Quartil & 5.0 & 9.0 \\
Média Aritmética & 4.0 & 7.1 \\
Desvio Padrão & 1.6 & 1.9 \\
Coef. de Variação & $40.1 \%$ & $27.1 \%$ \\
p-valor (comparação) & \multicolumn{2}{c}{$<0.0001^{*}$ ( $\left.\mathrm{t}=8.09\right)$} \\
p-valor (correlação linear) & $0.8454(\mathrm{r}=0.0304$ Correlação Inexistente) \\
\hline
\end{tabular}

Fonte: Autores, 2019.

\section{Desempenho no pós-teste 1 e no pós-teste 2}

Ao comparar o desempenho dos estudantes no pós-teste 1 e 2, objetiva-se comparar o aprendizado retido a curto e médio prazo (Tabela 2).

Observou-se significativa redução na média entre os testes: no pós-teste 1 , como já relatado, a média foi de 7.1 pontos e no pós-teste 2 caiu para 5.9 pontos, confirmado pelo teste t de Student $\left(p\right.$-valor $\left.<0.0001^{\star}\right)$. A correlação de Pearson para essas duas variáveis indicou real correspondência. 
Tabela 2 - Avaliação do Pós-teste e Pós-teste 2 de n=42 estudantes de medicina. Belém/PA, ano 2018.

\begin{tabular}{lcc}
\hline & $\begin{array}{c}\text { Pós-teste } \\
(\mathbf{n}=\mathbf{4 2})\end{array}$ & $\begin{array}{c}\text { Pós-teste 2 } \\
(\mathbf{n}=\mathbf{4 2})\end{array}$ \\
\hline Mínimo & 3.0 & 2.0 \\
Máximo & 10.0 & 9.0 \\
Mediana & 7.0 & 6.0 \\
Primeiro Quartil & 6.0 & 4.3 \\
Terceiro Quartil & 9.0 & 7.0 \\
Média Aritmética & 7.1 & 5.9 \\
Desvio Padrão & 1.9 & 1.9 \\
Coef. de Variação & $27.1 \%$ & $31.9 \%$ \\
p-valor (comparação) & \multicolumn{2}{c}{$<0.0001^{*}(\mathrm{t}=5.81)$} \\
p-valor (correlação linear) & $<0.0001^{*}(\mathrm{r}=0.7293$ Forte Correlação) \\
\hline
\end{tabular}

Fonte: Autores, 2019.

\section{Desempenho no OSCE-1 e OSCE-2}

No 1ํ OSCE se apresentou a média 7.7 com desvio padrão de 1.8. Já no $2^{\circ}$ OSCE se apresentou média 7.3 com desvio padrão de 1.2 .

Pelo teste $T$ de Student para amostras pareadas, com $p$-valor $=0.2225$, foi demonstrado que não houve real diferença nas notas obtidas entre o $1^{\circ}$ OSCE e o $2^{\circ}$ OSCE (Tabela 3 ).

Tabela 3 - Avaliação da correlação e da diferença entre as notas nos Pós-testes e nos OSCE. Amostra de $\mathrm{n}=42$ estudantes de medicina. Belém/PA, ano 2018.

\begin{tabular}{lll}
\hline & $\mathbf{1}^{\circ}$ OSCE & $\mathbf{2}^{\circ}$ OSCE \\
\hline Mínimo & 3.2 & 5.2 \\
Máximo & 9.8 & 9.3 \\
Mediana & 8.3 & 7.4 \\
Primeiro Quartil & 6.4 & 6.4 \\
Terceiro Quartil & 9.0 & 8.3 \\
Média Aritmética & 7.7 & 7.3 \\
Desvio Padrão & 1.8 & 1.2
\end{tabular}

Fonte: Autores, 2019. $\mathrm{p}$-valor $=0.2225$, teste $\mathrm{t}$ de Student para amostras pareadas.

\section{Correlação e diferença entre pós-teste e OSCE}

A Tabela 4 mostra a avaliação da diferença e da correlação entre as médias na primeira e na segunda etapa da pesquisa, considerando avaliação teórica (pós-teste) e prática (OSCE). Na avaliação da correlação entre $1^{\circ}$ Pós teste e 1을 OSCE, o valor foi significante, demonstrando significância estatística, situação semelhante ao encontrado na avaliação da correlação entre 2ํㅜ Pós-Teste e 2o OSCE (Tabela 4). 
Tabela 4: Avaliação da correlação e da diferença entre as notas nos Pós-testes e nos OSCE. Amostra de $\mathrm{n}=42$ estudantes de medicina. Belém/PA, ano 2018.

\begin{tabular}{lll}
\hline & $\mathbf{1}^{\circ}$ Pós-teste x $\mathbf{1}^{\circ}$ OSCE & $\mathbf{2}^{\circ}$ Pós-teste x $\mathbf{2}^{\circ}$ OSCE \\
\hline AVALIAÇÃO DA CORRELAÇÃO & & $r=0.6041$ \\
Coeficiente de Correlação & $r=0.3540$ & Regular correlação \\
Interpretação da correlação & Fraca correlação & $<0.0001^{*}$ \\
p-valor (Correlação) & $0.0214^{*}$ (significante) & \\
AVALIAÇÃO DA DIFERENÇA ENTRE AS MÉDIAS & 1.5 (Um ponto e meio) \\
Diferença média & 0.5 (meio ponto) & $<0.0001^{* *}$ (significante) \\
p-valor (diferença) & 0.0971 (não significante) &
\end{tabular}

Fonte: Autores, 2019. *Correlação linear de Pearson ${ }^{* *}$ Teste t de Student para amostras pareadas.

\section{Pós-teste 1 e OSCE- 1}

Foi observado 7,1 como a média do Pós-teste 1 e 7,7 como a média do OSCE- 1.

Foi observada real correlação entre as notas do $1^{\circ}$ Pós -teste e do $1^{\circ}$ OSCE, rejeitando-se, portanto a Hipótese Nula. Contudo, não houve real diferença entre as notas do $1^{\circ}$ Pós -teste e do $1^{\circ}$ OSCE, nesse caso, aceitando-se a Hipótese Nula (Gráfico 1).

Gráfico 1 - Avaliação da correlação e da diferença entre as notas no 1ํㅜㅇs-teste e o 1ํOSCE. Amostra de $n=42$ estudantes de Medicina. Belém/PA, ano 2018.

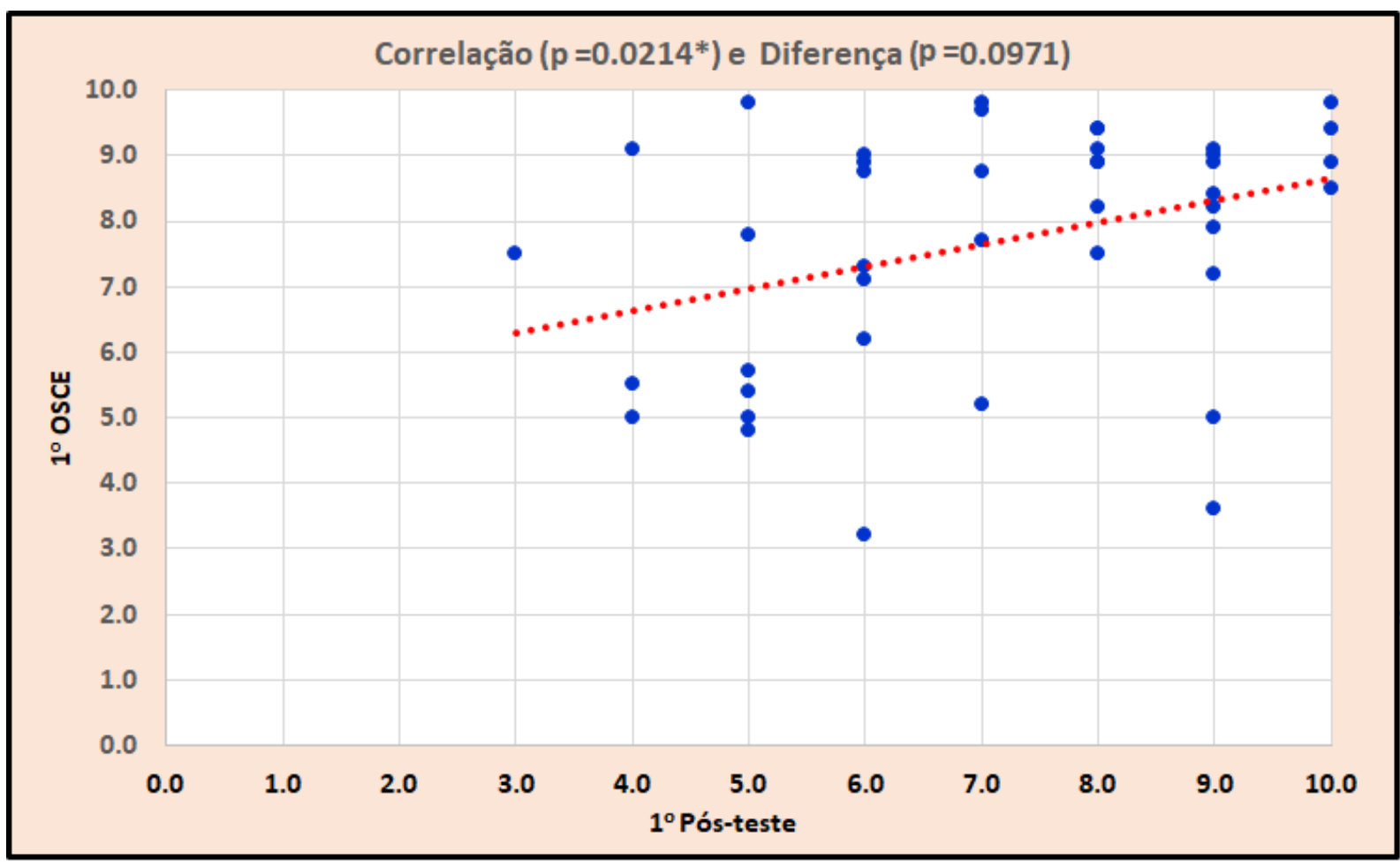

Fonte: Autores, 2019. 


\section{Pós-teste 2 e OSCE -2}

A média no Pós-teste 2 foi 5,9 e no OSCE-2 foi de 7,3. Foi observada correlação significativa entre as notas do pós -teste-2 e do OSCE-2. Dessa forma a Hipótese Nula foi rejeitada. Não obstante, foi constatada real diferença entre as notas do pós-teste-2 e do OSCE-2, rejeitando, portanto, a Hipótese Nula (Gráfico 2).

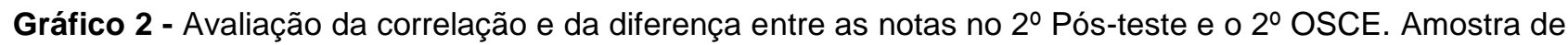
$\mathrm{n}=42$ estudantes de Medicina. Belém/PA, ano 2018.

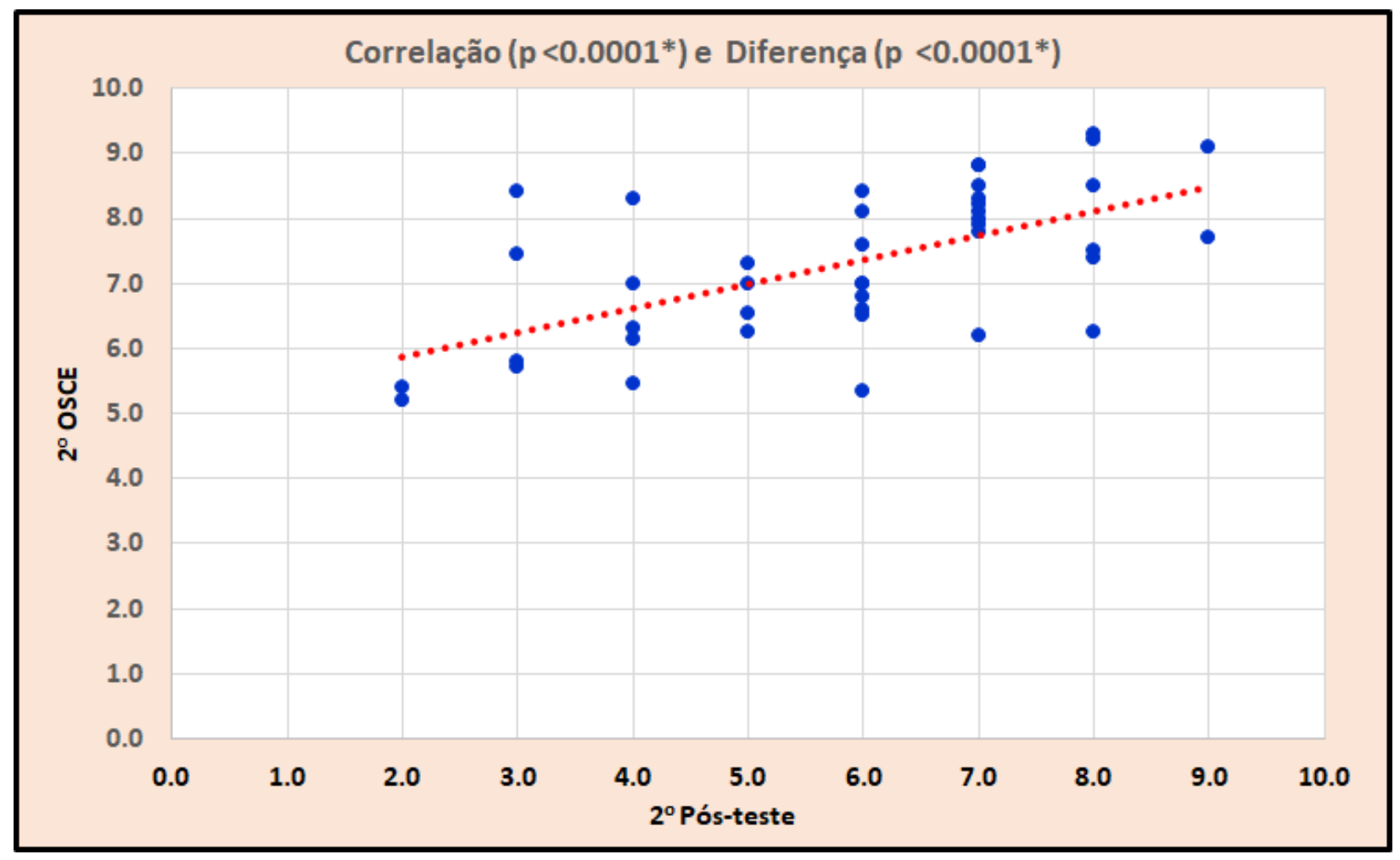

Fonte: Autores, 2019.

\section{DISCUSSÃO}

As habilidades clínicas têm papel importante no currículo dos profissionais de saúde, com um rol variado de técnicas e procedimentos avaliativos. Contudo, observa-se que os estudantes de Medicina ainda apresentam deficiências na aquisição dessas habilidades. (HOLMBOE, 2004).

Uma pesquisa recente realizada por Yang e colaboradores (2018), avaliou residentes de Medicina Intensiva antes e após um curso de simulação de alta fidelidade e encontrou resultados semelhantes, com pontuações superiores observadas no pós-teste teórico quando comparado ao pré-teste. Cenário semelhante também foi encontrado por Rogers e colaboradores (2001), que buscou avaliar o conhecimento de estudantes de Medicina utilizando 3 métodos avaliativos. Tanto nas avaliações teóricas quanto práticas, a pontuação no pós foi maior de forma significativa.

Já na avaliação comparativa entre o desempenho no pós-teste 1 e pós-teste 2, observou-se significativa redução na média entre os testes, com forte correlação estatística. Isso pode ser explicado pelo tempo decorrido entre as duas avaliações, período durante o qual os estudantes não tiveram novo contato, pela grade curricular, com temas ou práticas em neurologia e exame neurológico. 
Esses dados estão de acordo com os encontrados nos estudos de Doomernik e colaboradores (2017), no qual 165 estudantes de Medicina foram avaliados quanto ao seu conhecimento em anatomia humana em 2 momentos, com intervalo de 1 ano e meio entre os testes. Foi demonstrada pontuação significativamente menor na segunda avaliação.

Charalampopoulos e colaboradores (2016) analisou a retenção do conhecimento teórico e habilidades práticas de médicos e enfermeiros submetidos ao Curso Europeu de Suporte de Vida Pediátrico (EPLS), e constatou redução significativa do conhecimento teórico após 4 meses da realização do curso.

Na presente pesquisa, os resultados encontrados na comparação do desempenho dos OSCE's, diferentes dos encontrados na comparação dos testes teóricos 1 e 2, não apresentaram diferença estatisticamente significativa. Isso sugere que a retenção das habilidades clínicas sofreu pouca influência do tempo em que os alunos não foram mais expostos a semiologia neurológica, experiência oposta ao averiguado com os conhecimentos teóricos.

Esses dados concordam com os resultados obtidos no estudo de Charalampoulos (2016), citado anteriormente, que mostrou retenção satisfatória de algumas habilidades de um curso de suporte de vida pediátrico em médicos e enfermeira 4 meses após a realização do mesmo, mesmo com a redução da performance nos testes escritos.

Na primeira fase do estudo, a avaliação teórica (pós-teste 1) e a avaliação prática (OSCE-1) apresentaram forte correlação, porém não houve real diferença entre as notas. Esse resultado indica que o desempenho teórico e prático estavam no mesmo nível nessa fase da avaliação.

Esses achados vão de encontro aos de Yang e colaboradores (2018), que observaram que os residentes que tinham maiores pontuações no pós-teste teórico não necessariamente mostravam melhor performance nas práticas de simulação. No estudo de Rogers (2001) também foi observado que a melhora nas avaliações práticas não tinha correlação com boas pontuações nos testes escritos.

A segunda etapa da avaliação mostrou correlação significativa entre as notas do pós-teste- 2 e do OSCE2. Além disso, foi constatada diferença significativa entre as notas dessas avaliações. Dessa forma, é possível inferir que a prática superou a teoria, ainda que as médias tenham sido um pouco menores que no pós-teste1 e no OSCE-1.

Um estudo de Lukas, em 2012, avaliou a confiabilidade do desempenho dos alunos no OSCE durante o rodízio na Neurologia e determinou que a pontuação obtida teve correlação significativa com as notas dos atendimentos clínicos e do exame médico nacional, porém não houve correlação com as avaliações subjetivas dadas pelos preceptores. Isso sugere que o OSCE é uma medida mais confiável do desempenho estudantil que as notas lançadas pelos professores durante o rodízio na especialidade.

Diversos estudos internacionais demonstraram que o desempenho de estudantes de Medicina no exame físico neurológico mostrou-se mais fraco do que em outros campos da avaliação clínica. (PEITZMAN, 2015) (ROZE, 2016) (STEICHEN, 2015).

Schon e colaboradores (2002) pesquisou a percepção de 345 sujeitos (estudantes de Medicina e médicos generalistas) e atestou que, das especialidades médicas, a neurologia era percebida como a mais difícil de todas, por todos os indivíduos pesquisados. Nessa situação foram identificados 3 motivos principais: primeiramente pela necessidade de saber neuroanatomia e neurociência; em segundo lugar pelo tipo e qualidade do ensino que receberam; e em terceiro lugar pela complexidade do exame físico neurológico.

Analisando o que foi exposto acima, observa-se que os estudantes demonstraram um bom desempenho no aprendizado a curto prazo, com equivalência na esfera cognitiva e na esfera prática. Na avaliação do desempenho a médio prazo observou-se redução do conhecimento teórico, porém mantendo bom desempenho nas habilidades práticas. Esses achados são compatíveis com diversos outros trabalhos na literatura. 
Para a excelência do ensino médico, é primordial que haja integração dos domínios cognitivo e psicomotor, e que o aluno seja capaz de mobilizar o conhecimento em atitudes, com raciocínio lógico. A fim de minimizar o medo e a percepção negativa da especialidade Neurologia, deve-se procurar formas de simplificar o ensino dessa e de sua semiologia, tornando seu ensino interessante à maioria dos estudantes.

Como esse estudo foi conduzido em centro único, com número reduzido de sujeitos na amostra, investigações multicêntricas posteriores podem corroborar e certificar esses resultados, oferecendo novos dados a fim de melhorar o ensino e a forma de avaliar o aprendizado da semiologia neurológica.

\section{CONCLUSÃO}

Os resultados mostraram um incremento das médias ao compararmos pré-teste e pós teste-1, um bom desempenho no aprendizado a curto prazo, com equivalência na esfera cognitiva com os testes teóricos e na esfera prática, com o OSCE. Na avaliação do desempenho a médio prazo observou-se redução do conhecimento teórico, porém mantendo bom desempenho nas habilidades práticas.

\section{REFERÊNCIAS}

1. AL-WARDY NM. Assessment methods in undergraduate medical education. Sultan Qaboos Univ. Med. J. v.10, n.2, p.203-209, ago.2010.

2. CHARALAMPOPOULOS $\mathrm{D}$, et al. Theoretical knowledge and skill retention 4 months after a European Paediatric Life Support course. Eur. J. Emerg. Med., v. 23, n.1, p.56-60, feb.2016.

3. CYRINO EG, TORALLES-PEREIRA ML. Trabalhando com estratégias de ensino-aprendizado por descoberta na área de saúde: a problematização e a aprendizagem baseada em problemas. Cad. Saúde Pública., v.20, n.3, p. 780-788, mai-jun.2004.

4. DOOMERNIK DE, et al. Longitudinal retention of anatomical knowledge in second-year medical students. Anat. Sci. Educ., v.10, n.3, p.242-248, jun. 2017.

5. HOLMBOE ES. Faculty and the observation of trainees' clinical skills: problems and opportunities. Acad. Med., v.79, n.1, p.16-22, jan. 2004.

6. ISMAIL HM. Views of undergraduate students on objective structured clinical examination in neurology: a preliminar report. J. Family Community Med., v.8 69, n.3, p.79-82, sep.2001.

7. JOZEFOWICZ RF. Neurophobia: the fear of neurology among medical students. Arch. Neurol., v. 51, n.4, p. 328-9, apr. 1994.

8. KHAN MU, ALJARALLAH BM. Evaluation of modified essay questions (MEQ) and multiple choice questions (MCQ) as a tool for assessing the cognitive skills of undergraduate medical students. Int. J. Health. Sci. (Qassim), v. 5, n. 1, p. 3943, jan. 2011.

9. LUKAS RV, et al. Student assessment by objective structured examination in a neurology clerkship. Neurology., v.79, n .7, p.681-685, aug. 2012.

10. MILLER GE. The assessment of clinical skills/ competences/performance. Acad. Med., v.65, supplem 9, p.63-67, sep. 1990 .

11. PEITZMAN SJ, CUDDY MM. Performance in physical examination on the USMLE Step 2 clinical skills examination. Acad. Med., v.90, n.2, p.209-13, 2015.

12. ROGERS PL, et al. Quantifying learning in medical students during a critical care medicine elective: a comparison of three evaluation instruments. Crit. Care. Med., v. 29, n.6, p.1268-73, jun. 2001.

13. ROZE E, et al. "The Move", an innovative simulation-based medical education program using roleplay to teach neurological semiology: students' and teachers' perceptions. Rev. Neurol., v.172, n.4-5, p. 289-94, apr-may, 2016.

14. SAFDIEH JE, et al. Standardized patient outcomes trial (SPOT) in neurology. Med. Educ. Online., v.14, n.16, jan.2011.

15. SCHON F, et al. Is clinical neurology really so difficult? Neurol Neurosurg Psychiatry., v.72, n.5, p.557-9, may. 2002.

16. STEICHEN O, et al. Assessment of clinical observation skills of last year medical students. Rev. Med. Interne., v.36, n.5, p.312-8, 2015.

17. VUMA S, SA B. Evaluation of the effectiveness of progressive disclosure questions as an assessment tool for knowledge and skills in a problem based learning setting among third year medical students at The University of The West Indies, Trinidad and Tobago. BMC Res. Notes., 8:673, nov. 2015. doi: 10.1186/s13104-015-1603-0

18. YANG CW, et al. Application of high-fidelity simulation in critical care residency training as an effective learning, assessment, and prediction tool for clinical performance. Journal of the Formosan Medical Association. dec. 2018. Disponível em: https://doi.org/10.1016/j.jfma.2018.12.003 